\title{
Building quality mHealth for low resource settings
}

\section{Journal Article}

Author(s):

Ettinger, Kate M.; Pharaoh, Hamilton; Buckman, Reymound Y.; Conradie, Hoffie; Karlen, Walter (D)

Publication date:

2016

Permanent link:

https://doi.org/10.3929/ethz-b-000119383

Rights / license:

In Copyright - Non-Commercial Use Permitted

Originally published in:

Journal of Medical Engineering \& Technology 40(7-8), https://doi.org/10.1080/03091902.2016.1213906 
Ettinger, K. M., Pharaoh, H., Buckman, R. Y., Conradie, H., \& Karlen, W. (2016). Building quality mHealth for low resource settings. Journal of Medical Engineering \& Technology, 40(7-8), 431-443. https://doi.org/10.1080/03091902.2016.1213906

\title{
Building quality mHealth for low resource settings
}

Kate Michi Ettinger ${ }^{1}$, Hamilton Pharaoh ${ }^{2}$, Reymound Buckman ${ }^{3}$, Hoffie Conradie ${ }^{4}$, Walter Karlen ${ }^{5}$

\author{
${ }^{1}$ Mural Institute, San Francisco, USA \\ ${ }^{2}$ Physiotherapy Department, University of the Western Cape, Belleville, South Africa \\ ${ }^{3}$ Airbus Operations Gmbh, Hamburg, Germany \\ ${ }^{4}$ Ukwanda Rural Research Center, Stellenbosch University, Worcester, South Africa \\ ${ }^{5}$ Department of Health Sciences and Technology, ETH Zurich, Switzerland
}

\section{Abstract}

In low and middle income countries (LMIC), community health care workers (CHCW) are the primary point of care for millions of people. Mobile phone health applications (mHealth app) are the preferred technology platform to deliver clinical support to CHCW. In LMIC limited regulatory oversight exists to guide quality and safety for medical devices, including mHealth. During development of a mHealth app to assist $\mathrm{CHCW}$ with patient assessment and clinical diagnosis in rural South Africa, we applied human-centred design (HCD) and a bioethics consultation. The HCD approach enabled us to develop a mHealth app that responded to the needs and capacities of CHCW. The bioethics consultation prompted early consideration of safety concerns, social implications of our mHealth app, and our technology's impact on the CHCW-patient relationship. In this study, we found that combining a HCD approach with bioethics consultation improved the design quality and reduced safety concerns for our mHealth app. 


\section{Background}

Over the last 15 years, the United Nations Millennium Development Goals increased attention on health care improvement in low and middle income countries (LMIC). Several programmes introduced community health care workers ( $\mathrm{CHCW}$ ) as an effective way to expand access to health care for people in LMIC [1]. Other initiatives have built upon the successful CHCW model, including the UN's Millennium Villages project that in 2013 set a goal for one Million CHCW to expand access to health care for 650 Million people in LMIC [2]. With this rise in access to health care delivered by $\mathrm{CHCW}$ who go into the community to deliver care, attention has increasingly focused on how best to equip them with tools to improve their diagnostic and treatment capabilities [2]. The aim has been to leverage technology in order to provide a high level of care that can safely be delivered by the $\mathrm{CHCW}$.

\subsection{Mobile health as a daily tool for community health care workers}

Particularly in LMIC, information and communication technologies have been heavily developed in recent decades in order to accelerate regional development. This digital telecommunication infrastructure enabled leapfrogging traditional land-based telecommunication systems [3]. As a result, mobile phone based communication is widely used in LMIC. This widespread availability of mobile phones facilitated the idea for mobile phone-based applications (app) as a diagnostic and treatment support tool [4].

A recent study showed that a majority (57\%) of the introduced mobile health (mHealth) apps were for data collection [5]. In South Africa, the work of $\mathrm{CHCW}$ includes providing basic health services, supported self-care for patients, health promotion and education. Further, they provide medication adherence support for patients with tuberculosis and HIV/AIDS. This support to 
patients with chronic diseases is expected to reduce disease complications and prevent unnecessary hospital visits and admissions [6]. The Department of Health in the Western Cape province of South Africa is discussing expanding the competences of $\mathrm{CHCW}$ by introducing new tasks such as screening for immunisations and providing maternal/child health care.

We developed ClinicalGuide, a mHealth app designed for use by $\mathrm{CHCW}$ in rural South Africa [7]. ClinicalGuide integrates the pneumonia diagnostic tree of the Integrated Management of Childhood Illnesses (IMCI) recommended by the World Health Organization (WHO) [8]. The IMCI guidelines have shown to be effective in primary healthcare clinics in the Western Cape province of South Africa [9]. Until recently, IMCI guidelines were only available in printed form, which made them difficult to navigate, hard to keep up-to-date and expensive to distribute.

\subsection{Developing a high quality system for low-resource markets}

Increasingly, evidence shows that health technologies are designed but not adopted due to the inappropriateness of technology or the lack of its usability; many mHealth apps are built, prototyped, but never used [5], [10]. While mobile phone use is ubiquitous throughout LMIC, this does not necessarily translate into $\mathrm{CHCW}$ adopting this technology in their daily work.

Manufacturers and innovators are often confronted with unclear regulatory guidelines in LMIC markets [11]. The standard method for addressing quality and safety for medical product design is to follow the regulatory standards of national governing bodies, such as the USA's Food and Drug Administration (FDA). Medical device regulations are developed on a national level and due to this path-dependent development, they are inconsistent worldwide [12].

This project focused on developing a high quality diagnostic tool for CHCW in LMIC. Thus, we applied human-centred design (HCD) methods in the development process to improve the usability and the likelihood of its adoption. Furthermore, to fulfil our commitment to build high 
quality apps in the face of the regulatory gap, we partnered with a bioethics consultant to have a health care ethics consultation (HCEC) from the outset of our design process. Bioethics, a discipline at the intersection of law, medicine and ethics, considers the legal, social and ethical implications of health technology, medicine and science on society [13]. This novel approach provided an opportunity to review the ethical considerations of the app and to better understand its acceptability.

Our objective was to evaluate the impact of both HCD and a HCEC on the development of a mHealth app for $\mathrm{CHCW}$ in South Africa. This manuscript describes how these interventions were used and their influence on the app's development process.

\section{Methods}

We introduced two methodological interventions at two stages of the app development process: the concept development stage and the prototype testing stage. The first intervention was the use of HCD to create a responsive app with good quality interaction- a user can easily and effectively navigate the interface and the device is useful and desirable. The second intervention consisted of a HCEC to identify and address ethical concerns, particularly related to safety of the app.

\subsection{Human-centred design}

HCD is a cadre of methods and processes that focus product design on the needs and capabilities of the intended user [14]. HCD involves observing, interviewing, and latent needs finding in order to understand the problem from the user's perspective. HCD counters the tendency to build product solutions in abstraction. HCD integrates feedback from prospective users at each stage of design. HCD informs solutions that are responsive to the user's experience and needs throughout a product's development. 


\subsubsection{Concept stage}

ClinicalGuide was developed iteratively in a user-driven approach with frequent, informal interactions between many stakeholders such as engineers, software programmers, $\mathrm{CHCW}$ and public health researchers. Engineers and software developers visited CHCW settings in South Africa over several iterations, evaluated needs and were immersed in the context. Following these steps, specifications for ClinicalGuide were developed and updated. The software was developed using an agile approach using Scrum with weekly sprints [15]. Sprints were planned using a Backlog that featured direct feedback from stakeholders in the field.

\subsubsection{Prototype stage}

Once a functional prototype was ready, we were interested in a formal usability evaluation. The primary goal of this evaluation was to assess three components of usability that would evaluate HCD effectiveness: 1) the efficiency of the app, 2) user satisfaction, and 3) the difficulty in learning to use the app.

ClinicalGuide was assessed in a usability study. We conducted a workspace analysis with structured interviews and observations in a simulated environment. The participants were asked to 'Think Aloud' [16], and an audio and video recorder logged the results. This technique is a wellestablished method to test user interfaces [17]; it enabled us to accurately track the interaction of each participant with the app and, thus, allowed us to assess for inefficiencies or deficiencies in the interface. Each participant was given two simulated patient scenarios to walk through using the assistance of a smartphone with the ClinicalGuide installed (Appendix A). Steps used and difficulties to assess the virtual patient were observed by a moderator (WK, HP). The moderator provided assistance if tasks could not be performed after two attempts. After finishing each scenario, the moderator interviewed the participant about difficulties encountered, actions that would have been done differently without the ClinicalGuide and general comments regarding the 
experience. User satisfaction was assessed with the Computer System Usability Questionnaire (CSUQ) [18]. Specific durations of each task, error rates and subjective feedback were used to identify usability difficulties.

\subsection{Health care ethics consultation}

In advanced care settings, a clinical ethics consultation may be requested when a clinician encounters two potential courses of action, each with different risks, burdens and benefits [19]. A clinical ethics consultation facilitates clinicians and patients to make shared decisions in these complex situations [20].

Introduction of mHealth technology enables the $\mathrm{CHCW}$ to deliver a higher level of care and broadens the scope of practise. However, as $\mathrm{CHCW}$ are isolated at the point of care, relying upon a digital assistant to guide clinical decisions, we recognized that the locus of clinical power shifts from trained clinicians in the clinic to $\mathrm{CHCW}$ in the field. Thus, developers need to understand the health care ethics implications of their technology on the clinician-patient relationship.

The consultation for ClinicalGuide addressed these aspects of health care ethics:

1) privacy, quality, reliability and safety of the technology

2) research with human subjects

3) clinical ethics issues arising from the technology

4) social justice implications with inclusive innovation considerations

At the time of the study, there were no health care ethics frameworks or assessments for mHealth apps targeting LMIC. Therefore, we developed a framework to consider the ethical implications of ClinicalGuide (See Appendix B). The ethics consultation framework drew upon: bioethics [13], research ethics [21], clinical ethics [19], [20], [22], ethics of care [23], public health ethics [24], human rights [25] and inclusive innovation [26]. 


\subsubsection{Concept stage}

The bioethics consultant (KE) applied the framework in an interview with the development team lead (WK). The interview was audio recorded and analysed. Issues that were identified during the consultation were highlighted for review with other members of the development team.

\subsubsection{Prototype stage}

The bioethics consultant applied the framework in interviews with the development team (WK, HP). Furthermore, in order to understand the ethical and relational implications of the new technology on shared decision making and the clinical relationship at the prototype usability stage, the bioethics consultant applied the framework in interviews with users (CHCW), a clinic nurse supervisor, and rural South African community members (i.e. potential patients). Interviews with $\mathrm{CHCW}$ and the clinic nurse supervisor were the same participants as the usability testing. These interviews were voice recorded without personal identification to maintain anonymity. The interviews followed a structured format (Appendix C) that began with open-ended questions eliciting the participant's experience with ClinicalGuide. Questions then probed the types of clinical uncertainties that $\mathrm{CHCW}$ regularly encountered and how $\mathrm{CHCW}$ responded to these difficult clinical situations. The interviews ended with questions that systematically assessed the CHCW perception of the benefits, risks and concerns of using ClinicalGuide in this setting. The recordings were transcribed and analysed. These interviews with $\mathrm{CHCW}$, patients and development team were summarized, analysed for issues, and discussion points were reported to the development team lead. 


\subsection{Subjects and study location}

After approval by the Health Research Ethics Committee 2 of Stellenbosch University, we had informal interviews with \# subjects at LOCATION during the concept stage. Then at prototype stage, $10 \mathrm{CHCW}$ were recruited and provided written informed consent for the usability analysis and ethics consultation in the prototype stage. We considered 10 a sufficiently large number for two study sites to identify the core issues in ClinicalGuide [27]. The subjects were active CHCW who work for the Home Community-Based Care programmes in Avian Park, Worcester and Rusthof Home Care, Paarl. The study was performed in a dedicated room at the headquarters of the respective centre. Additionally, two community members and a nursing supervisor were recruited and provided consent to participate in this study.

\subsection{Materials}

ClinicalGuide prompts the $\mathrm{CHCW}$ to ask relevant clinical questions, prompts for observed symptoms, provides recommendations and suggests treatments. ClinicalGuide automatically logs assessment data and records administered treatments, which are saved to a local electronic database. For the usability study, ClinicalGuide was customized to the workflow of the CHCW in the field. It included a Tuberculosis Direct Observation Therapy Short-course (TB DOTS) rule set that presents a series of instructions for drug administration as well as a questionnaire for drug side effects. The WHO IMCl cough and difficult breathing rules of Western Cape's Department of Health were implemented. They consisted of observation and questions regarding general danger signs, followed by specific questions regarding pneumonia diagnosis. Treatment for pneumonia did not include prescription and administration of antibiotics as this was outside the scope of $\mathrm{CHCW}$ in this district at the time. 
ClinicalGuide was installed on two low-to middle range Android smartphones (Samsung Galaxy Nexus and HTC One V). At the time of the study both phone models were more than a year old and functionality matched recent low-cost smartphones. The two models were chosen for their variation in screen size (Nexus: 4.65 inches, $720 \times 1280$ pixels; One V: 3.7 inches, $480 \times 800$ pixels) allowing for testing the usability of the interface in two configurations.

\section{Results}

\subsection{Concept Stage}

\subsubsection{Human-centred design}

Observations and interviews showed that $\mathrm{CHCW}$ visit 1-20 assigned patients per day, mostly travelling by foot. Collecting data for each patient is a heavy burden for $\mathrm{CHCW}$, because various administrative entities require different documentation for each patient. Not all CHCW are trained in the same manner and they do not all have the same medical proficiencies. Due to the distances travelled, as well as the absence of their supervisor while visiting their clients at home, many $\mathrm{CHCW}$ are disconnected from the health system at the point of care. Mobile phones are available and people frequently use smartphones that feature a large touch screen. Therefore, the integration of electronic guidelines into a mHealth app is possible.

Analysis of the weekly time investment revealed that administration, transportation and patient care take up equal portions of the available time. Therefore, administrative features such as generation of statistics and weekly reports were developed and included into the app. These reports were configured so that they could be adapted to the varying reporting requirements of the care centres. 
Immobile chronic disease patients were reported to be the most frequently visited patients. Encounters with sick children were rare. The IMCl guidelines were not frequently needed nor applied. Although $\mathrm{IMCl}$ were part of the training, $\mathrm{CHCW}$ did not show proficiency on all aspects and did not carry the guidelines with them. These observations supported our efforts to digitize the $\mathrm{IMCl}$ guidelines.

\subsubsection{Ethical considerations}

The systematic review during the concept stage facilitated identifying ethical issues proactively. Our framework included the relational aspects of the technology- how the technology would impact the clinical relationship, the social implications of the $\mathrm{CHCW}$ using this new technology and the cultural gaps between the developer team and the users.

The interview reviewed technical aspects of the app, such as who might be adversely impacted by hardware/software failures, what measures were taken to mitigate those failure risks, and how would failures/updates be managed. It also assessed the clinical aspects of ClinicalGuide, such as whose clinical guidelines were selected, how would clinical guidelines be maintained/updated and how would the device impact the $\mathrm{CHCW}$-patient relationship. The social implications of the new technology were considered; giving someone a smartphone might be a motivator to adopt the new technology, it might elevate their status in the community and/or it might make them a target for crime. There were no right or wrong answers. The framework prompted discussion that fostered awareness of these potential issues early enough so that they could be addressed in the design process. 
Reviewing these questions at the outset of the design allowed the development team to consider including social and ethical implications in the specifications and requirements. The team found that having these issues raised strengthened usability and supported better design, because they were prompted to see beyond the technical impact of the app and were able to better appreciate the social context of the $\mathrm{CHCW}$ and patients.

\subsection{Prototype Stage}

\subsubsection{Human-centred design}

\section{Participants}

Six participants from the study site in Paarl and four from Worcester enrolled in the usability study. The participants were all female and between 24 and 50 years old. They had between 3 months and 17 years of health care experience. All participants personally owned a mobile device; participants used mobile phones frequently (6) or occasionally (4). Four participants owned a smartphone (touchscreen) and used this frequently; the same number of participants had never used a smartphone. All ten participants indicated inexperience using personal computers (PC); six had never used a PC.

\subsubsection{Usability}

In scenario A, four tasks were not completed a single time each (Figure 1). The incomplete tasks A11-13 were skipped because the subject mistakenly pressed the 'home' button during task A11 and the scenario was stopped early. Observing this behaviour prompted us to add a warning popup to confirm the user's desire to end an assessment prematurely. The login task (A2) required the highest rate of help from the moderator, due to difficulties in understanding touch screen keyboard activation. 
In scenario B, the task that caused the most difficulties was B4 'using the calendar function' (Figure 2). Other incomplete tasks were creating a new patient (one participant) and closing the application (one participant).

The median time to complete scenario A was $6.9 \mathrm{~min}$ (range $5.5-10.6 \mathrm{~min}$ ) and was $6.8 \mathrm{~min}$ (range 4.1 -12 $\mathrm{min}$ ) for scenario B. Self-reported inexperienced smartphone users performed the slowest assessments in scenarios $A$ and $B$, and experienced smartphone users completed the two scenarios fastest.

A perceived challenge was creating a new patient record from the patient management screen. The icon in the upper right corner of the screen was not identified immediately as a possible entry point. Overall, the participants found that not all icons were intuitive; though they also commented that once the icons were explained, the use of the ClinicalGuide was much easier. One participant confused the Yes/No input and inverted the answers given during scenario A. This went unnoticed in the subsequent review screen. However, since an incorrect diagnosis was suggested by the app, the participant overrode the proposed incorrect diagnosis and an alternative, more likely diagnosis was chosen. In three scenarios, participants overrode the suggested diagnosis/treatment. From debriefing, the reasons for these override decisions included one accidental and two intentional due to a perceived incorrect diagnosis.

Participants using the smartphone with the smaller screen size commented on the fact that the font size was very small and found it difficult to read at times. It was further stated that typing using the small letters and numbers of the smartphone keyboard was difficult.

The patient assessment process was judged to be easy to use. It was commented that the process of the assessment was easier and quicker than the traditional paper based assessment. Statements were: 'Quicker than writing down', or 'Less writing', 'Collect information easier and quicker. Not lose information'. 
[Insert Figure 1 Here]

[Insert Figure 2 Here]

\subsubsection{Computer system usability questionnaire (CSUQ)}

CSUQ analysis showed a high degree of overall satisfaction with the use of the ClinicalGuide on the smartphone. An average score of 2.96 out of 3 was obtained; where 3 would be complete satisfaction and -3 no satisfaction. The five questions that did not receive full scores (2) were:

- It was simple to use this interface.

- I feel comfortable using this interface.

- It was easy to learn to use this interface.

- This interface gives error messages that clearly tell me how to fix the problems.

- The information (such as the manual, on-screen messages, and other documentation) provided with this interface is clear.

One comment obtained was 'It was a bit difficult at the beginning but the more you work with this interface, it is going to be a useful thing'.

\subsubsection{Health care ethics consultation}

\subsubsection{Interviews with development team}

The interview questionnaire of the team at the prototype stage resulted in no new issues being identified. However, the results of the 14 user interviews at this stage provided useful and important feedback and insights for the development team. 


\subsubsection{Interviews with $\mathrm{CHCW}$}

The depth of training (range: three months to one year) and experience were evenly distributed across both sites. Generally, $\mathrm{CHCW}$ reported a high degree of cooperation from patients and their families. Nonetheless, all $\mathrm{CHCW}$ had encountered at least one difficult situation including navigating conflicts between patient preferences and clinical recommendations (particularly for behavioural/lifestyle changes related to chronic disease) and being asked to do tasks beyond their scope of care. As well, $\mathrm{CHCW}$ often mediate conflicts between patients and their family members regarding care. $\mathrm{CHCW}$ regularly contact their nursing supervisor when they encounter a situation about which they were uncertain - this was done by making a phone call from the patient's home or discussing the case upon return to the clinic.

CHCW reported positively about their initial experience with ClinicalGuide and its implications for their workflow. They did not have concerns about privacy of data, because they already collect patient data on paper. None believed that their patients would object to them using the app or their data privacy, and four recognized that they would have to explain the app to their patients in order for them to feel comfortable about it. Two participants raised concerns about the reliability focusing on what happens to data if the phone would crash, the phone was stolen or the network was down. Since the use of a backup function was not explained as part of the usability study, we considered this as indicating that the $\mathrm{CHCW}$ valued the importance of the data collected.

$\mathrm{CHCW}$ were confident that if they were provided a mobile device from their health care centre, then it would mean that the device had been adequately vetted for quality. At least two participants expressed that it was imperative for the app to explain the basis for its recommendations in order for them to rely upon it.

CHCW had varying perspectives on whether they would trust recommendations of the app when 
these departed from their own judgment. Five responded that they would do what the app prompted while three said that they would likely trust their own judgment over the app and two said they would contact their supervisor. This variation did not correlate with length of training or years of experience. When asked about the safety of the app and whether it might bring harm to patients, two $\mathrm{CHCW}$ raised concerns about their own personal safety carrying a smartphone in the areas that they serve.

\subsubsection{Interviews with patients}

Two community members, who could be prospective patients with ClinicalGuide, were interviewed. All were amenable to the idea that a $\mathrm{CHCW}$ might use a smartphone to gather information and guide clinical decision making. They did not express any concerns regarding privacy, quality, reliability or safety of the device. Generally, they exhibited a deferential view toward the $\mathrm{CHCW}$ as a health care provider. However, when posed with the hypothetical question about their child being very sick but the device suggested to wait for two days, all said that they would take the child to the clinic/hospital against the advice of an app.

\subsubsection{Impact of Interventions}

[Insert Table 1 Here]

HCD provided the significant insights that impacted the product's design and development; likewise, the HCEC with the development team raised awareness and impacted the specifications and design at the concept stage. However, at the prototype stage, the HCEC with the users ( $\mathrm{CHCW}$ ) provided the most significant insights to improve the product's design. 


\section{Discussion}

In this study we showed that the use of a smartphone equipped with a diagnostic application can serve as a work aid for CHCW in rural South Africa. By applying HCD methods and a HCEC, we identified usability problems, user concerns and safety issues that might otherwise not be identified prior to implementation.

\subsection{Human-centred design aspects}

Very little is known about the usability of mHealth systems in low resource settings, in particular for $\mathrm{CHCW}$. In Hudson et al. a pulse oximeter for the smartphone was successfully evaluated for usability in Uganda [28]. However, the study focused on use in the operating room by trained anaesthesia personnel. In a similar approach, a predictive app for post-discharge deaths in Ugandan hospitals was assessed for usability. Subsequently, implemented improvements led to shorter task completion times of data entry and vital sign measurement [29]. IMCI have been implemented into personal digital assistants and tested for efficiency and compared to standard practice of trained clinicians in Tanzania [30]. Overall, 27 clinicians increased their adherence to $\mathrm{IMCl}$ guidelines. A mHealth tool for decision support on cardiovascular disease was developed in collaboration with end-users and field tested in India [31]. All three of these studies were conducted with trained medical personnel and thus, may not directly apply to CHCW. In an Ethiopian maternal health setting it was reported that $\mathrm{CHCW}$ rapidly learned the use of touch screens and were comfortable with mHealth apps [32]. However, this was assessed only during the implementation phase without formal usability testing during prototype development. 
It was suggested that mHealth tools can easily become a burden to health workers if the solution is too technologically focused [33]. It was pointed out that particularly for $\mathrm{CHCW}$ for whom the only prerequisite is literacy and who serve as the frontline of health care delivery for many people in Africa, the expansion of skills and competencies via an electronic device should include evaluation of usability. Mobile phones as functional job aids can improve the likelihood of adoption by $\mathrm{CHCW}$ [33]. This aligns with our goal to provide functional and usable mHealth solutions that could reduce the administrative work burden of $\mathrm{CHCW}$. As previously discussed by Kallander et al., apps should be tailored to the target population and users should be included in the design process in order to prevent negative impact of technology on the workload of CHCW [10].

Technology should not only be used to facilitate data collection, but should support the CHCW in efficiently accomplishing tasks. Reports of CHCW training and capacities are varied [34]. Thus, it was unclear how prepared $\mathrm{CHCW}$ were to use smartphone-based clinical apps. When developing ClinicalGuide, we applied HCD methods because we recognized the importance of understanding the clinical and technical capacities of our intended users. Our HCD analysis revealed that CHCW spend a third of their time with administration and reporting. Thus, there was a need for an app that enabled electronic record keeping. Participants acknowledged the potential value of recording assessments electronically. We believe that we have reached a suitable compromise between complexity and usability of the app. Data entry was limited to the entry of new patient information, which in the case of the $\mathrm{CHCW}$ working in a home based environment, does not occur frequently. In a different setting where $\mathrm{CHCW}$ would encounter many new patients, this could become a significant time burden and would need to be studied separately. The CSUQ usability questionnaire revealed very high acceptance with no rating below 2 out of 3 , where 3 is 
the highest satisfaction and -3 the lowest. Interestingly, this high acceptance coincides with observations in other mHealth usability studies [29], [31].

\subsection{Ethical and safety aspects}

Early identification of ethical concerns means that these issues can be addressed in the design process. While mHealth app developers face many constraints, every design decision reflects an exercise of discretionary power. The pressure to rapidly develop a working prototype at minimal cost is intense. Unlike medical devices, where a product is completed, then tested for efficacy, mHealth apps tend to be developed iteratively, released with bugs and updated regularly even after they are in use. The 'fail early, fail often' mantra touted in design and agile technology development may not be appropriate when applied to health care -- in health care, when we fail, we fail real people. In this regard, mHealth apps differ critically in their development from traditional medical devices [35]. At the time this project was initiated, the FDA was silent about regulation of mHealth apps [36], and there was no regulatory guidance in the local South African market for mHealth apps. Thus, our ethics consultation framework was designed to sensitize the mHealth app development team to the ethical and relational impact of their technology. Our development team felt that reflecting on these considerations early in concept development was helpful to inform specifications and requirements.

In clinical settings, ethical dilemmas for clinicians and patients often arise around decision making due to the imbalance of power inherent in clinical relationships. A patient is vulnerable and dependent on the clinician to accurately diagnose and prescribe appropriate treatment. Bioethics serves as a guide to balance the power in clinical interactions by balancing the principles to honour

a person's autonomy (respect for persons), to promote patient well-being (beneficence) and to do no harm (non-maleficence) [13]. CHCW reported encountering ethical dilemmas such as being 
asked to provide care beyond their scope of practise and negotiating conflicts with patients about adhering to medication plans. $\mathrm{CHCW}$ recognized the need to balance their clinical interest in a patient's wellbeing (beneficence) with the patient's right to self-determination (autonomy). $\mathrm{CHCW}$ recognized their patients' rights and the importance of partnering with their patients for shared decisions about care. $\mathrm{CHCW}$ reported negotiating these difficult situations adeptly, and as needed, they sought the support of their nursing supervisor.

For the ClinicalGuide development team, it was informative to have heightened awareness of the clinical relationship dynamics, the shared decision making model, and the need to balance competing bioethical principles. As mHealth apps, such as ClinicalGuide, expand the scope of practise for $\mathrm{CHCW}$ enabling $\mathrm{CHCW}$ with low levels of training to fulfil higher skilled clinical tasks, it becomes essential for developers to understand the ethical implications of their technology on the clinical relationship. To improve the likelihood that $\mathrm{CHCW}$ will follow a mHealth app recommendation, mHealth apps that respond to the variability among $\mathrm{CHCW}$ users could provide the rationale for a recommendation, confirm whether the user followed the app's recommendation, and evaluate clinical outcomes to enhance confidence in its recommendations. Further, tracking the frequency and type of recommendations overruled could lead to improved decision rules and to identifying topics for further training.

Given that regulatory oversight in LMIC is limited [11], yet CHCW expect that a device provided to them will have met quality standards, mHealth app developers have a significant responsibility to deliver a well-designed and safe product. Particularly for mHealth apps developed outside of traditional academic settings (i.e. without institutional ethics review board oversight), a systematic review of key ethical considerations for the app, prototype testing and the implementation strategy would be beneficial. A recently proposed method for systematic identification of ethical aspects of health technology in Sweden provides an additional resource 
for developers [37]; however, this framework was developed in/for Sweden; it has a more theoretical approach and expects access to an ethicist, it does not address the relational implications of technology, and it does not contemplate the culture gaps often in LMIC contexts. For ClinicalGuide, our interventions, with a focus on how the technology impacted relationships, yielded insights that improved the app's design and acceptability. In light of our positive experience with these complementary interventions (HCD and HCEC), we would like to promulgate the concept of relation-centred design [38]. Integrating these two methods into a single framework that addressing power dynamics and relational implications of new technology on the clinical relationship. Relation-centred design prompts mHealth app developers to build technology that strengthens the clinical relationship, attends to its inherent power imbalances and enhances collaborative decision making.

We found that the relationship between developers and users has parallels to the power imbalances in the clinician-patient relationship, because users are dependent upon developers to design products, in which users can trust that they will be able to fulfil their clinical obligations reliably. Thus, many of the ethical implications that we considered between a provider and patient could also be applied to the relationship between the developer and the user. Particularly, the inclusive innovation questions to 'mind the gap' could be applied to the culture gap between the development team (highly educated urban dwelling) and the users (low resource settings) where considerable regional, cultural and socio-economic variation was present. Thus, systematically addressing ethical issues throughout the product design process may raise developers' ethical sensitivity to the app as well as their own role.

\subsection{Limitations}

Limitations included potential for bias in our data collection. The app developer conducted the 
usability interviews, and the HCEC interviews were conducted by the designer of that intervention. Thus, there is a potential for bias since the same person designed the intervention, executed the interviews and analysed the findings. To mitigate this bias, the interviews were recorded for impartial review. In the future, having an independent interviewer complete the interviews could mitigate this issue. Although the HCEC framework was developed from a synthesis of existing and established ethics consultation methods, this case study was the first time that the framework was applied.

At the prototype stage, the sample size representing a limited geographic region of the Western Cape was small given that the 10 users were divided between two sites. Also, the patient interviews were conducted with a self-selected group living in rural South Africa served by CHCW, but was too small a sample size to draw generalizable conclusions.

\subsection{Conclusion}

In this study, we found that the combination of a HCD approach with a HCEC yielded complementary insights to improve the app and to reduce safety concerns for a mHealth app designed for $\mathrm{CHCW}$ in rural South Africa.

ClinicalGuide holds promise for reducing time spent on administrative tasks. We gained insight about $\mathrm{CHCW}$ practise and their perspectives on technology trustworthiness. Identified concerns, such as data reliability and quality of clinical recommendations, raised awareness in the development team.

From the earliest stage of mHealth app development, proactively addressing ethical considerations helped to inform design decisions, influenced specifications for the app, and raised the ethical sensitivity of the design team. The use of ethics consultation allowed for the identification of potential risks from the introduction of digital assistants that would not have 
emerged with usability and workspace analysis alone. Therefore, including these health care ethics considerations early in the design process of mHealth apps and usability testing, particularly in LMIC markets, can be informative.

As mHealth apps increasingly inform clinical decision making, we recommend that mHealth app developers apply the complementary methods of HCD and a HCEC framework into their development process.

\section{$5 \quad$ Acknowledgements}

We would like to thank to all the $\mathrm{CHCW}$ that participated in this study. The authors would like to thank all staff of Ukwanda Rural Health Centre of University of Stellenbosch in Worcester and Rusthof home-care in Paarl, South Africa, for their keen interest and input to the design of ClinicalGuide and Wayne Swart, Erin Cooke and Isabel Chan who assisted with transcribing the ethics consultation interviews. Our gratefulness also belongs to the late Prof. Cornie Scheffer who always supported our work.

\section{Declaration of Interest section}

The authors have no conflicting interests. Kate Ettinger is a bioethics consultant at Mural institute and founder of the non-commercial, open source initiative, OpenQRS (http://www.openqrs.org/). Walter Karlen has published ClinicalGuide as open source project (https://github.com/ecemgroup/clinicalguide). Reymound Buckman is a freelance research consultant for Syte, a private e-health consulting firm. 


\section{References}

[1] A. Liu, S. Sullivan, M. Khan, S. Sachs, and P. Singh, "Community health workers in global health: scale and scalability.," Mt Sinai J Med, vol. 78, no. 3, pp. 419-35, 2011.

[2] P. Singh and S. Sullivan, One Million Community Health Workers: Technical Task Force Report. The Earth Institute, Columbia University, 2013.

[3] J. C. Aker and I. M. Mbiti, "Mobile Phones and Economic Development in Africa," J Econ Perspect, vol. 24, no. 3, pp. 207-32, 2010.

[4] Vital Wave Consulting, mHealth for Development: The Opportunity of Mobile Technology for Healthcare in the Developing World. Washington, D.C. and Berkshire, UK: UN Foundation - Vodafone Foundation Partnership, 2009.

[5] R. Braun, C. Catalani, J. Wimbush, and D. Israelski, "Community health workers and mobile technology: a systematic review of the literature.," PLoS One, vol. 8, no. 6, p. e65772, Jan. 2013.

[6] J. Volmink and P. Garner, "Systematic review of randomised controlled trials of strategies to promote adherence to tuberculosis treatment," BMJ, vol. 315, no. 7120, pp. 1403-6, Nov. 1997.

[7] W. Karlen and C. Scheffer, "Design of an interactive medical guideline application for community health workers.," in Annual International Conference of the IEEE Engineering in Medicine and Biology Society., 2014, pp. 1366-1369.

[8] WHO, IMCl: Caring for Newborns and Children in the Community - Manual for the Community Health Worker, 5th ed. Geneva, CH: World Health Organization, 2011.

[9] M. Chopra, S. Patel, K. Cloete, D. Sanders, and S. Peterson, "Effect of an IMCl intervention on quality of care across four districts in Cape Town, South Africa.," Arch Dis Child, vol. 90, no. 4, pp. 397-401, 2005.

[10] K. Källander, J. K. Tibenderana, O. J. Akpogheneta, D. L. Strachan, Z. Hill, A. H. a ten Asbroek, L. Conteh, B. R. Kirkwood, and S. R. Meek, "Mobile health (mHealth) approaches and lessons for increased performance and retention of community health workers in low- and middle-income countries: a review.," J Med Internet Res, vol. 15, no. 1, p. e17, 2013.

[11] K. M. Ettinger, "Open Issues and a Proposal for Open Data Monitoring to Assure Quality, Reliability and Safety in Health Care Devices Targeting Low and Middle-Income Countries," in Technologies for Development. What is Essential?, S. Hostettler, E. Hazboun, and J.-C. Bolay, Eds. Lausanne, CH: Springer International Publishing, 2015, pp. 81-90.

[12] C. Altenstetter, "Medical device regulation in the European Union, Japan and the United States . Commonalities, differences and challenges," vol. 25, no. 4, pp. 362-389, 2012.

[13] T. L. Beauchamp and J. F. Childress, Principles of biomedical ethics. New York: Oxford University Press, 2001.

[14] Human Centred Design Toolkit, 2nd ed. IDEO, 2013.

[15] K. Schwaber and M. Beedle, Agile Software Development with Scrum. Prentice Hall PTR, 2001.

[16] M. W. Van Someren, Y. F. Barnard, and J. A. C. Sandberg, "The think aloud method: A practical guide to modelling cognitive processes," in Information Processing \& Management, vol. 31, no. 6, San Diego: Academic Press, 1994, pp. 906-7.

[17] A. W. Kushniruk, M. M. Triola, E. M. Borycki, B. Stein, and J. L. Kannry, "Technology induced error and usability: the relationship between usability problems and prescription errors when using a handheld application.," Int J Med Inform, vol. 74, no. 7-8, pp. 519- 
26, Aug. 2005.

[18] J. J. R. Lewis, "IBM computer usability satisfaction questionnaires: psychometric evaluation and instructions for use," Int J Hum Comput Interact, vol. 7, no. 1, pp. 57-78, 1995.

[19] B. Lo, Resolving Ethical Dilemmas: A Guide for Clinicians, 5th ed. Philadelphia: Lippincott Williams \& Wilkins, 2013.

[20] L. F. Post, J. Blustein, and N. Dubler, Handbook for Health Care Ethics Committees. Baltimore: Johns Hopkins University Press, 2006.

[21] C. Coleman, J. Menikoff, J. Goldner, and N. Dubler, The Ethics and Regulation of Research with Human Subjects. San Francisco CA: Matthew Bender \& Company, 2005.

[22] A. R. Jonsen, M. Siegler, and W. J. Winslade, Clinical Ethics: A Practical Approach to Ethical Decisions in Clinical Medicine, 7th ed. McGraw-Hill, 2010.

[23] C. Gilligan, J. Ward, J. M. Taylor, and B. Bardige, Mapping the Moral Domain A Contribution of Women's Thinking to Psychological Theory and Education. Cambridge: Harvard University Press, 1989.

[24] S. Anand, Fabienne Peter, and A. Sen, Public health, ethics, and equity. Oxford: Oxford University Press, 2004.

[25] P. Farmer, "Challenging orthodoxies: The road ahead for health and human rights Paul Farmer," vol. 10, no. 1, pp. 1-11, 2008.

[26] K. M. Ettinger, "Mind the gaps," Blog post, 2008. [Online]. Available: http://www.ideasthatimpact.org/ideas-that-impact/minds-the-gaps-scribd. [Accessed: 25-Nov-2015].

[27] J. Nielsen and T. K. Landauer, "A mathematical model of the finding of usability problems," in Proceedings of the SIGCHI conference on Human factors in computing systems - CHI '93, 1993, pp. 206-13.

[28] J. Hudson, S. M. Nguku, J. Sleiman, W. Karlen, G. A. Dumont, C. Petersen, C. B. Warriner, and J. M. Ansermino, "Usability testing of a prototype Phone Oximeter with healthcare providers in high- and low-medical resource environments," Anaesthesia, vol. 67, no. 9, pp. 957-67, 2012.

[29] L. L. English, D. Dunsmuir, E. Kumbakumba, J. M. Ansermino, C. P. Larson, R. Lester, C. Barigye, A. Ndamira, J. Kabakyenga, and M. O. Wiens, "The PAediatric Risk Assessment (PARA) Mobile App to Reduce Postdischarge Child Mortality: Design, Usability, and Feasibility for Health Care Workers in Uganda," JMIR mHealth uHealth, vol. 4, no. 1, p. e16, 2016.

[30] B. DeRenzi, G. Borriello, N. Lesh, T. Parikh, C. Sims, W. Maokla, M. Chemba, Y. Hamisi, D. Hellenberg, and M. Mitchell, "E-imci: improving pediatric health care in low-income countries," in Proceeding of the twenty-sixth annual CHI conference on Human factors in computing systems - CHI '08, 2008, pp. 753-62.

[31] A. Raghu, D. Praveen, D. Peiris, L. Tarassenko, and G. Clifford, "Lessons from the Evaluation of a Clinical Decision Support Tool for Cardiovascular Disease Risk Management in Rural India," in Technologies for Development. What is Essential?, S. Hostettler, E. Hazboun, and J.-C. Bolay, Eds. Springer International Publishing, 2015, pp. 199-209.

[32] A. Little, A. Medhanyie, H. Yebyo, M. Spigt, G.-J. Dinant, and R. Blanco, "Meeting community health worker needs for maternal health care service delivery using appropriate mobile technologies in Ethiopia.," PLoS One, vol. 8, no. 10, p. e77563, 2013.

[33] D. L. Strachan, K. Källander, A. H. A. Ten Asbroek, B. Kirkwood, S. R. Meek, L. Benton, L. Conteh, J. Tibenderana, and Z. Hill, "Interventions to improve motivation and retention 
of community health workers delivering integrated community case management (iCCM): stakeholder perceptions and priorities.," Am J Trop Med Hyg, vol. 87, no. 5 Suppl, pp. 111-9, Nov. 2012.

[34] Z. A. Bhutta, Z. S. Lassi, G. Pariyo, and L. Huicho, Global Experience of Community Health Workers for Delivery of Health Related Millennium Development Goals: A Systematic Review, Country Case Studies, and Recommendations for Integration into National Health Systems. Global Health Workforce Alliance, World Health Organization, 2010.

[35] C. J. Vincent, G. Niezen, A. A. O'Kane, and K. Stawarz, "Can Standards and Regulations Keep Up With Health Technology?," JMIR mHealth uHealth, vol. 3, no. 2, p. e64, 2015.

[36] W. Garvin, "The Legal Perspective of mHealth in the United States," J Mob Technol Med, vol. 1, no. 4, pp. 42-45, 2012.

[37] E. Heintz, L. Lintamo, M. Hultcrantz, S. Jacobson, R. Levi, C. Munthe, S. Tranæus, P. Östlund, and L. Sandman, "FRAMEWORK FOR SYSTEMATIC IDENTIFICATION of ETHICAL ASPECTS OF HEALTHCARE TECHNOLOGIES: THE SBU APPROACH," Int J Technol Assess Health Care, vol. 31, no. 3, pp. 124-130, 2015.

[38] K. M. Ettinger, "Ethical Considerations and Relation-Centered Design for Mobile Health Applications," in 2014 EPFL - UNESCO Chair International Conference on Technologies for Development, Lausanne, CH: Cooperation \& Development Center (CODEV), EPFL, 2014. 


\section{Appendix A: \\ Think Aloud protocol, scenarios (Table A1) and matching pathway (Figure A1)}

\begin{tabular}{|l|l|}
\hline Task & Description \\
\hline Scenario A & Tuberculosis Direct Observation Therapy Short-course (TB DOTS) \\
\hline & \\
\hline A1 & Start Clinical Guide \\
\hline A2 & Login to system \\
\hline A3 & Start Follow up \\
\hline A4 & Select patient \\
\hline A5 & Start new assessment \\
\hline A6 & Select DOTS \\
\hline A7 & Enter side effects (ASK) \\
\hline A8 & Enter side effects (LOOK) \\
\hline A9 & Administrate Medicine \\
\hline A10 & Review Assessment \\
\hline A11 & Select recommendation \\
\hline A12 & Confirm recommendation \\
\hline A13 & Select treatments \& finish assessment \\
\hline & \\
\hline Scenario B & IMCl cough and difficult breathing \\
\hline & \\
\hline B1 & Start new assessment \\
\hline B2 & Create new patient \\
\hline B3* & Using Calendar \\
\hline B4 & Save new patient data \\
\hline B5 & Start IMCI Cough \& Difficult breathing \\
\hline B6 & Enter Danger Signs (ASK) \\
\hline B7 & Enter Danger Sign (LOOK) \\
\hline B8 & Enter IMCl (ASK) \\
\hline B9 & Enter IMCl (LOOK, LISTEN, FEEL) \\
\hline B10 & Review Assessment \\
\hline B11 & Select recommendation \\
\hline B12 & Confirm recommendation \\
\hline B13 & Close application \\
\hline B14 & \\
\hline Table A1: List of tasks for the Think Aloud scenario A (TB DOTs) and scenario B (IMCl Cough \& Difficult Breathing). \\
\hline
\end{tabular}




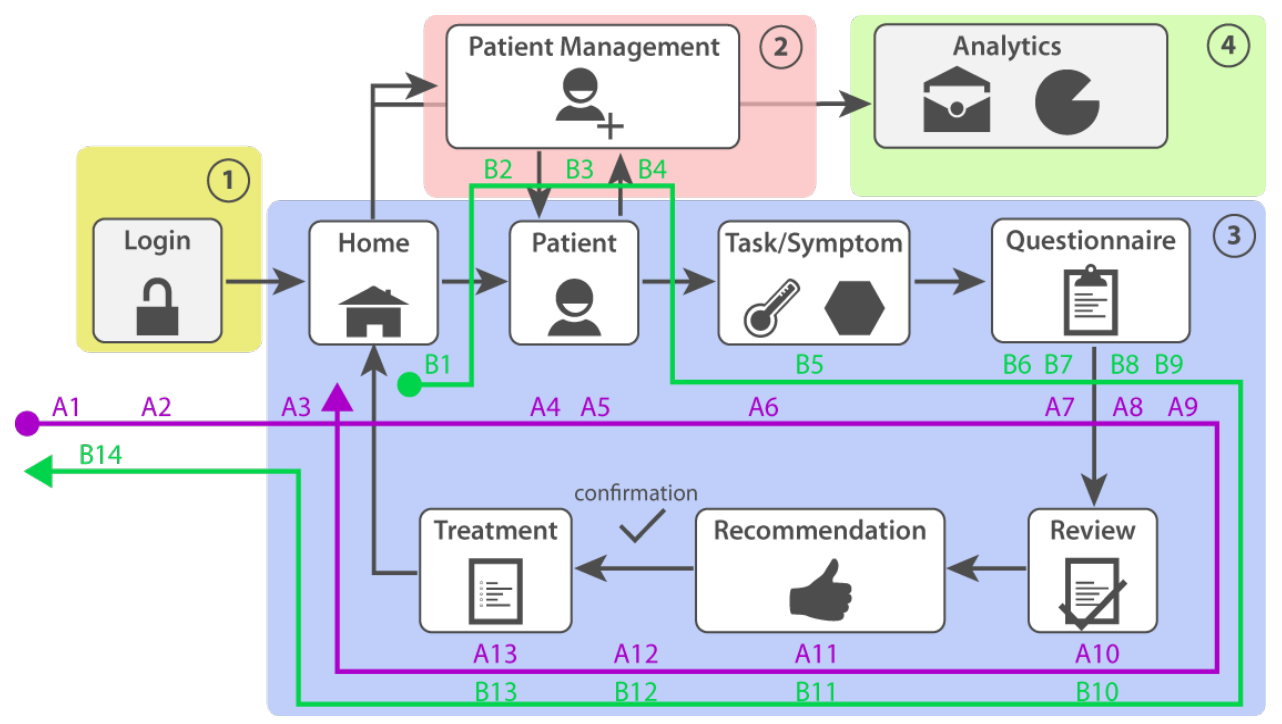

Figure A1: Schematic of work flows possible in the ClinicalGuide. The application is composed of 4 main modules (1 Login/user admin; 2 Patient management; 3 Patient assessment workflow; 4 Analytics (not tested in this study)). The coloured arrows show the pathway for the two Think Aloud scenarios. Purple: Scenario A is a TB DOTS follow-up assessment where an existing user is being visited. Green: Scenario B is a young child that is assessed for cough and difficult breathing and requires first entry in the database. 


\section{Appendix B: Health care ethics considerations for mHealth apps developers}

To be reviewed during each stage of the app development process. Many of the ethical implications that we consider between a provider and patient can be translated to how we think about the relationship between the developer and the user. These questions can be applied to review the technical implications as well as the clinical outcomes.

\section{Five key ethical considerations (PQRST):}

1) Is privacy $(P)$ of data an issue with this mHealth app? How will it be handled? What will be done if there is a breach of the privacy? On your system's end? If the device gets stolen? What harms could come to patient users if this data is compromised? How does this patient community feel about the privacy of their health data? Will there be a way to accommodate variations in preferences among patients regarding the privacy of their data?

2) How will you assure the quality (Q) of this mHealth app? What are concerns about quality for this mHealth app that you need to address? What would the outcomes be of a false positive? Of a false negative? How will you monitor for that feedback ? What will be the criteria to change the algorithms? What amount of scientific data will justify a change the protocol algorithms? How will you keep the app updated? Will you let users know when you update the app?

3) How will you assure the reliability $(\mathrm{R})$ of this mHealth app? What is the worst harm that could come from the app failing? How will you track when the app crashes? What will be your response?

4) How will you assure a patient's safety (S) with this mHealth app? What are the adverse outcomes that could occur as a result of this app? How will you mitigate those safety concerns?

5) How do you intend to address transparency (T) with regard to issues that may arise with this mHealth app? Will you disclose bugs identified and crash reports? app updates? algorithm changes? If so, how will you do this? If not, why would you choose not to do this? What will you do to address the trustworthiness of your mHealth app?

\section{Ethical considerations when working with people (potential users) for prototype testing:}

1) Who will be the users of this mHealth app? Is this a vulnerable group? What, if any, additional considerations need to be addressed with this user group (e.g. children, prisoners, etc.)? Who will be the test subjects during the prototype phase? What are the criteria for their selection/exclusion? How will you recruit people to participate? Why is that the best approach? Is there an aspect of recruitment that may be coercive?

2) How will you get the participant's informed consent? Who will explain the consent process to them? What will that person say? Are there concerns about people potentially not having the capacity to consent or the freedom to refuse? Does the person overseeing the informed consent process know how to assess for decision making capacity ? Is there an aspect of this consent process that may be coercive? 
3) What are the benefits of the mHealth app? What are the risks of the mHealth app? Do the benefits outweigh the risks? If a harm arises from participating in the prototype study, how will you address/redress that? Do the participants fully appreciate the risks?

4) How are you assessing the mHealth app's validity? How many people do you need to show that the app is safe and effective? Is this prototype study designed effectively to achieve the needed outcome? Is this prototype study designed in the least invasive way for the human subject?

5) Are there any conflicts of interest among the team (recruiters, researchers, developers) with the app? With the people who will provide informed consent and the subjects? Any other potential conflicts of interest?

Many mHealth apps may not be required to go through institutional/ethics review board (IRB/ERB). In countries where there are no regulatory mechanisms, this framework provides an important minimum foundation to assure ethical integrity in one's mHealth app development.

\section{Clinical Ethics Considerations: Relation-Centred Design and Inclusive Innovation:}

1) How does the clinical relationship factor into the problem as identified?

2) How does each participant in the clinical relationship view the problem as identified?

3) How does this concept for mHealth app influence the clinical relationship?

4) How does shared decision making fit into this app's function? Is it necessary? desirable ?

5) How does the app support shared decisions?

6) What are the gaps of culture between clinician and patient?

7) What are the gaps of geo-political-socio-economics between clinician and patient?

8) What are the gaps of systems between clinician and patient?

9) What are the gaps of power between clinician and patient?

10) How does the app address these gaps to bridge and/or minimize them?

For mHealth app developers who design apps for people from a difference place, such as an mHealth app developed in North America for use in Africa, the last four minding the gap questions that address the relational differences between the clinician and the patient may also be relevant to consider the culture gaps between the mHealth app developer and the users. These mind the gap questions identify four relational areas to consider for inclusive innovation when developing a new technology. 


\section{Appendix C: \\ Questions to elicit health care ethical concerns of $\mathrm{CHCW}$}

\section{Scope of Practise}

1. What types of patients do you see?

2. What was your training?

3. How long have you been in your current role as $\mathrm{CHCW}$ ?

4. How does your practise work?

a. Do you see the same patients regularly?

b. What is your schedule?

c. Do you see patients in their homes or in the clinic or both?

\section{Ethical Encounters in Practise}

1. Do you ever encounter situations where you are uncertain about what to do for a patient when you are in the field?

a. Please describe some examples.

b. How often does this happen?

c. What do you do when a situation like this arises?

2. Please describe if you ever encounter situations that made you feel uneasy or uncomfortable when you are in the field and what you did?

3. Please describe if you ever had a conflict with a patient or their family about the care plan and what you did?

4. Do you ever have conflicts with other $\mathrm{CHCW}$ over how to provide care for a patient?

5. What is your familiarity with the concept of health care ethics? If so, how do you understand it in your work?

a. In the care provider relationship, you have a power imbalance because the patient is dependent on you. How do you handle this power?

b. What if what you want for a patient is different from what s/he wants, how do you resolve those situations?

\section{Experience with ClinicalGuide}

1. How was your experience with the device?

2. Do you think that it will help you seeing patients? (If so, how?)

3. Do you think this device will change how you interact with patients?

4. Do you have any concerns about using this device?

5. Do you think your patients will have any concerns about you using this device?

6. Do you have any concerns about having the patient's data on it?

7. Do you have any concerns about privacy for the patient's data?

8. Do you have any concerns about the reliability of the device?

9. Do you have any concerns about the quality of the recommendations?

10. Do you have any concerns about the safety for the patients from you using this device?

11. Are you concerned about your own safety carrying this smartphone device?

12. Hypothetical: If there was a case where the app said that there is no pneumonia, but when you looked at the child with your eye, the child looked very sick, would you follow the app's recommendation? 


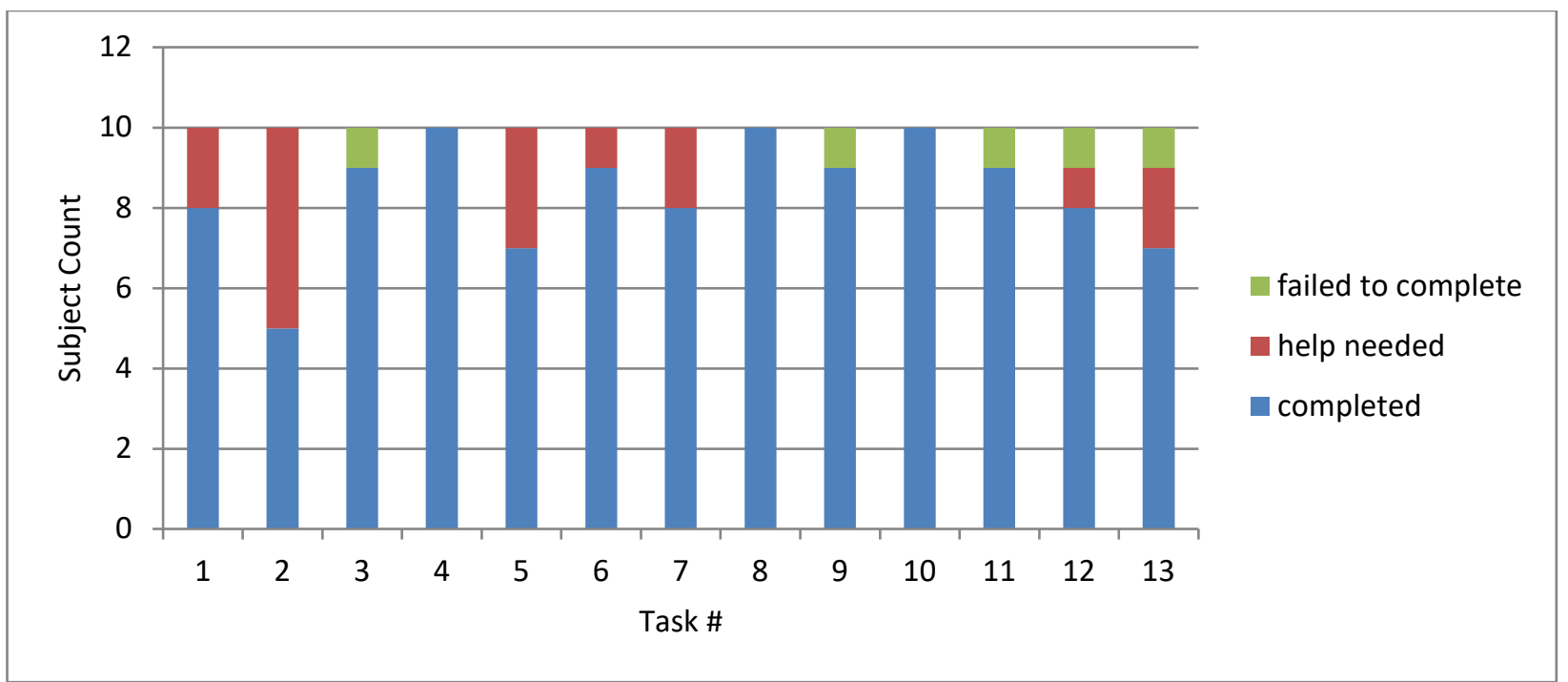

Figure 1: Completion rates for each task in scenario A. See Table A1 for task descriptions. 


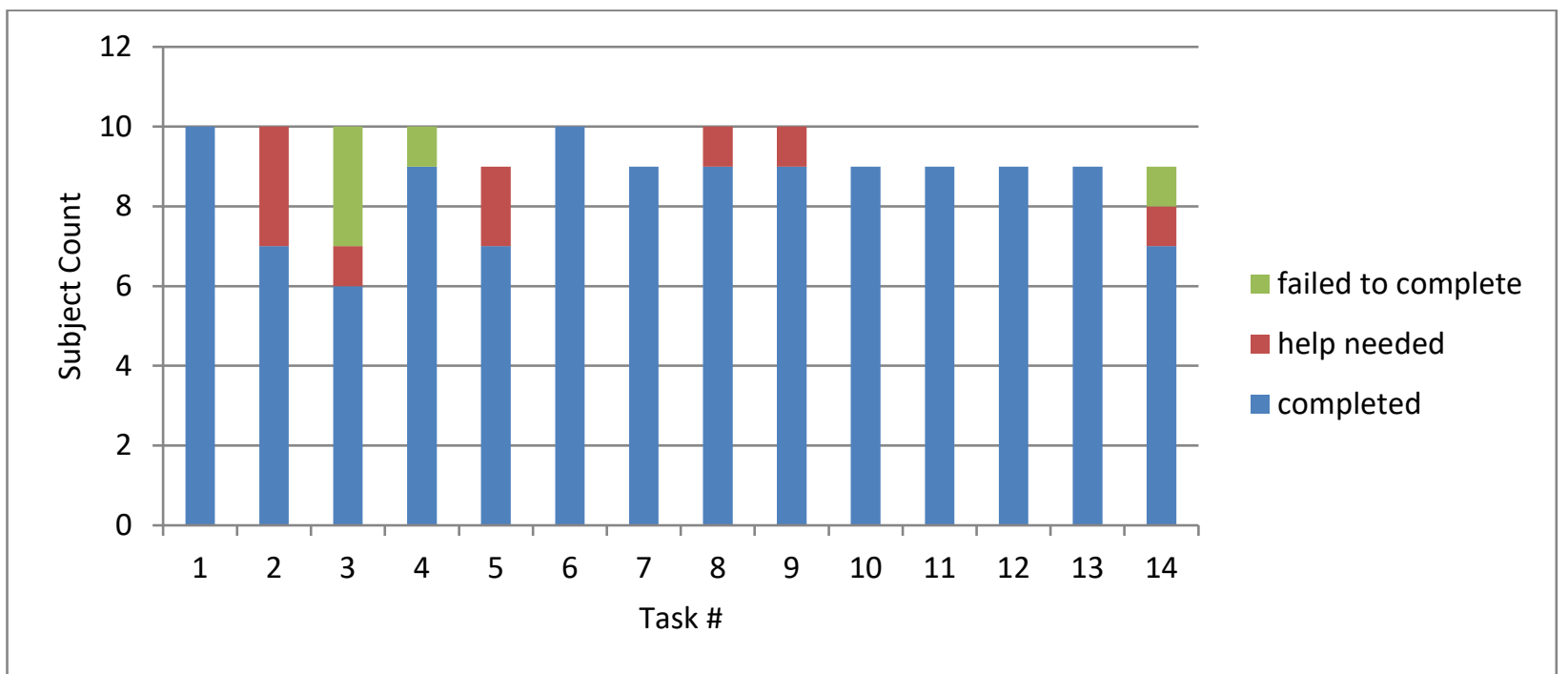

Figure 2: Completion rate for each task in scenario B. See Table A1 for task descriptions. 
Table 1. Main impact of human-centred design (HCD) and Ethics Interventions

\begin{tabular}{|c|c|c|c|}
\hline Stage & Impact Awareness & $\begin{array}{c}\text { Impact on } \\
\text { Specifications }\end{array}$ & Impact on Design \\
\hline \multicolumn{4}{|c|}{ Concept Stage } \\
\hline HCD & $\begin{array}{ll}\text { - } & \text { High admin } \\
& \text { burden } \\
\text { - } & \text { Easy travel } \\
\text { - } & \text { Little IMCI } \\
& \text { expertise } \\
\end{array}$ & $\begin{array}{l}\text { - Inclusion of job } \\
\text { aid function } \\
\text { - } \\
\text { Provision of } \\
\text { decision support }\end{array}$ & $\begin{array}{ll} & \text { Fast workflow } \\
\text { - } & \text { Task logging for } \\
& \text { reporting } \\
\text { - } & \text { Secondary learning } \\
& \text { features } \\
\end{array}$ \\
\hline $\begin{array}{l}\text { Ethics Interviews } \\
\text { (developers) }\end{array}$ & $\begin{array}{l}\text { - } \quad \text { Social status } \\
\text { change of } \mathrm{CHCW} \\
\text { - } \quad \text { Failures. Who is } \\
\text { impacted? } \\
\text { - } \quad \text { New regulations }\end{array}$ & $\begin{array}{l}\text { - } \text { Modular } \\
\text { approach for fast } \\
\text { changes cultural } \\
\text { adaptations }\end{array}$ & $\begin{array}{l}\text { - } \text { Results review } \\
\text { - Confirmation dialogs for } \\
\text { critical decisions }\end{array}$ \\
\hline \multicolumn{4}{|c|}{ Prototype Stage } \\
\hline $\begin{array}{l}\text { HCD: } \\
\text { Usability }\end{array}$ & $\begin{array}{l}\text { - Inappropriate } \\
\text { icons }\end{array}$ & $\begin{array}{ll}\text { - } & \text { Increase screen } \\
\text { size } \\
\text { - } & \text { Additional } \\
\text { labelling for } \\
\text { review screen }\end{array}$ & $\begin{array}{l}\text { - } \quad \text { Reduction of text entry } \\
\text { - } \\
\text { - } \quad \text { Colour label for yes /no } \\
\text { - } \quad \text { Complement icons with } \\
\text { - } \quad \text { Simplification on data } \\
\text { reporting }\end{array}$ \\
\hline $\begin{array}{l}\text { HCD: } \\
\text { User Satisfaction }\end{array}$ & - High acceptance & $\begin{array}{l}\text { - Adaptive learning } \\
\text { functions }\end{array}$ & $\begin{array}{l}\text { - Provide better learning } \\
\text { instructions }\end{array}$ \\
\hline $\begin{array}{l}\text { Ethics Interviews } \\
\text { (CHCW) }\end{array}$ & $\begin{array}{l}\text { - Safety of data } \\
\text { and CHCW }\end{array}$ & $\begin{array}{l}\text { - Add encryption } \\
\text { and adapted } \\
\text { backup solutions }\end{array}$ & $\begin{array}{l}\text { - Minimize need for high- } \\
\text { end devices }\end{array}$ \\
\hline $\begin{array}{l}\text { Ethics Interviews } \\
\text { (Patients) }\end{array}$ & $\begin{array}{l}\text { Patients showed } \\
\text { high trust } \\
\text { towards } \mathrm{CHCW} \\
\text { and also devices }\end{array}$ & & \\
\hline
\end{tabular}

